\title{
Multifaceted and mixed didactic-interactive methods are more effective ways to learn and change behaviour
}

\author{
Abstracted from \\ Firmstone VR, Elley KM, Skrybant MT, Fry-Smith A, Bayliss S, Torgerson CJ. \\ Systematic review of the effectiveness of continuing dental professional \\ development on learning, behavior, or patient outcomes. J Dent Educ 2013; 77: 300-315. \\ Address for correspondence: Dr Carole J. Torgerson, School of Education, \\ Durham University, Leazes Road, Durham, DH1 1TA, UK. Email: carole.torgerson@durham.ac.uk
}

\section{Question: Is continuing dental professional development effective?}

Data sources The following electronic databases were searched from 1986 to the present: Cochrane Library (Cochrane Database of Systematic Reviews, DARE and HTA Databases, and Cochrane CENTRAL Register of Controlled Trials), Medline In Process and Other NonIndexed Citations and Medline (Ovid), Embase (Ovid), ERIC (CSA), Index to Theses (Expert Information Services), and ZETOC (British Library Electronic Table of Contents and conference proceedings). No language restrictions.

Study selection Randomised controlled trials or quasi-experimental designs were eligible which evaluated continuing professional development (CPD) interventions in dentistry (for dentists and professions complimentary to dentistry) on learning gains, behaviour change or patient outcomes. These could be compared against no intervention or another CPD intervention. There were no minimum follow up times.

Data extraction and synthesis The following data were extracted by two reviewers: bibliographic details, settings, characteristics about participants, intervention and control conditions, outcome measures, findings and conclusions. Studies were grouped thematically according to the intervention, and the studies were quality assessed using a custom system based on the CONSORT statement. The results were reported in a narrative form and no attempt was made to combine them in a meta-analysis.

Results Ten studies were included. Eight studies were judged to be of high to moderately high quality. None of the studies measured patient outcomes, but a multifaceted black box intervention resulted in selfreported patient care and face-to-face contact to change antibiotic prescribing habits showed a change in clinician behaviour. The size of this effect for both of these is not reported here. There was some effect on knowledge but not on behaviour for courses and workshops, written material, computer-assisted learning and audit and feedback. Conclusions Multi-method and multi-phased dental CPD has potential for the greatest impact on practitioner behaviour.

\section{Commentary}

This systematic review had a clear aim, namely to 'to synthesize existing evidence evaluating the effectiveness of continuing professional development (CPD) interventions in dentistry on learning gains, behavior change, or patient outcomes.' They took a broad definition of what CPD could include though they ultimately identified studies that included courses/workshops, written information, computer-aided learning, audit/self-reflection, face-to-face support, and 'black box' combinations of CPD interventions.

There are a couple of quibbles one might have with the search strategy, the choice of appraisal method and the absence of figures to indicate the magnitude of effect where there was one, but the overall conclusions seem reasonable.

As with reviews of various interventions to change all health professionals' behaviour conducted by the Cochrane EPOC group, ${ }^{1}$ the results are broadly not too encouraging. I think, though, that we should be careful in drawing too many conclusions from the findings of this review not through any fault of the authors but because of the paucity of studies they could include. However, the narrower EPOC review of education meetings alone suggests that even where more studies are included the effect of continuing education may be moderate with a risk difference (RD) of $6 \%$ (interquartile range 1.8 to 15.9 ) in compliance with desired practice for those who attend. ${ }^{2}$ In that review there was no effect on behaviour change where the behaviour was a complex one but there was a greater improvement when didactic and interactive methods were used (median adjusted RD 13.6).

There is much discussion in the knowledge translation literature about the importance of the context a clinician works within when designing interventions to change behaviour, ${ }^{3}$ something that a one-off lecture or seminar will struggle to incorporate. It may be that the face-to-face intervention reported in this review that did appear to change clinician behaviour was able in part to do this. Knowledge may be a necessary component of change, but not a sufficient one.

Of course it is possible that the picture may be a little brighter and that clinicians do indeed change behaviour but not in the way the researchers measured it. There can be a naïve assumption that knowledge is simply transferred to clinicians and put into practice. Adult learning theories came to undermine this idea, recognising that adults come with their own experiences and make of the knowledge they encounter what they will. Should we expect dentists to behave differently from any other adult learners? Thus they may learn from the CPD but in ways not anticipated, transform- 
ing the knowledge into something useful to them. Researchers in this field may need to develop more nuanced and sensitive ways of evaluating the effect of CPD, perhaps using qualitative rather than quantitative methods.

A final comment here arises from ethnographic studies that have indicated that our minds as clinicians do not appear to work in the rationalistic way that some would like to think and that learning is more socially-determined through encounters with both explicit and tacit knowledge. ${ }^{4}$ There are many reasons why we may or may not consciously or subconsciously use new knowledge in our practice $^{5-7}$ So if CPD is to be more effective than the current reviews suggest, we may need to reconsider the way in which we deliver it, thinking more about the 'after sales service' of facilitating the incorporation of new learning into clinicians' daily lives. A recent review of facilitation to increase adoption of clinical guidelines in general medical practice found that practices where this happened were 2.76 (95\% CI, 2.18-3.43) times more likely to adopt the guidelines. ${ }^{8}$ Might CPD events need a facilitation bolt-on to make them more effective?

\section{Practice point}

- Multifaceted and mixed didactic-interactive methods in CPD are more effective ways to learn and change behaviour.
Dominic Hurst

Department of Adult Oral Health, Barts and The London School of Medicine and Dentistry, Queen Mary University of London, London, and Department of Primary Care Health Sciences, University of Oxford, Oxford, UK

1. Cochrane Effective Practice and Organisation of Care Group. 2012 [updated 16/10/2012; cited 2013 23/02/2013]. Available from: http://epoc.cochrane.org/.

2. Forsetlund $L, B j o r n d a l ~ A$, Rashidian $A$, et al. Continuing education meetings and workshops: effects on professional practice and health care outcomes. Cochrane Database Syst Rev 2009; 15: CD003030. PubMed PMID: 19370580.

3. McCormack B, Kitson A, Harvey G, Rycroft-Malone J, Titchen A, Seers K. Getting evidence into practice: the meaning of 'context'. J Adv Nurs 2002; 38: 94-104. PubMed PMID: 11895535.

4. Gabbay J, le May A. Evidence based guidelines or collectively constructed "mindlines?" Ethnographic study of knowledge management in primary care. Br Med J 2004; 329(7473): 1013. PubMed PMID: 15514347. Pubmed Central PMCID: 524553

5. Dopson S, FitzGerald L, Ferlie E, Gabbay J, Locock L. No magic targets! Changing clinical practice to become more evidence based. Health Care Manage Rev 2010; 35: 2-12. PubMed PMID: 20010008. Epub 2009/12/17. eng.

6. Cabana MD, Rand CS, Powe NR, et al. Why don't physicians follow clinical practice guidelines? A framework for improvement. JAMA 1999; 282: 1458-1465. PubMed PMID: 10535437. Epub 1999/10/27. eng.

7. Hopper L, Morris L, Tickle M. How primary care dentists perceive and are influenced by research. Community Dent Oral Epidemiol 2011; 39: 97-104. PubMed PMID: 21091753. Epub 2010/11/26. eng.

8. Baskerville NB, Liddy C, Hogg W. Systematic review and meta-analysis of practice facilitation within primary care settings. Ann Fam Med 2012; 10: 63-74. PubMed PMID: 22230833. Pubmed Central PMCID: 3262473.

Evidence-Based Dentistry (2013) 14, 67-68. doi:10.1038/sj.ebd.6400944 